Supplement of Arch. Anim. Breed., 59, 59-64, 2016

http://www.arch-anim-breed.net/59/59/2016/

doi:10.5194/aab-59-59-2016-supplement

(C) Author(s) 2016. CC Attribution 3.0 License.

(c) (i)

Supplement of

\title{
Genetic diversity of domesticated and wild Sudanese guinea fowl (Numida meleagris) based on microsatellite markers
}

\section{Weimann et al.}

Correspondence to: G. Erhardt (georg.erhardt@agrar.uni-giessen.de)

The copyright of individual parts of the supplement might differ from the CC-BY 3.0 licence. 
Table S1: Characteristics of the polymorphic microsatellites: Annealing temperature (AT), $\mathrm{MgCl}_{2}$ concentration, size (bp) and number of alleles.

\begin{tabular}{|l|l|l|l|l|}
\hline Locus & $\mathrm{AT}\left({ }^{\circ} \mathrm{C}\right)$ & $\mathrm{MgCl}_{2}(\mathrm{mM})$ & Size (bp) & Number of \\
& & & & alleles \\
\hline MCW222 & 51.0 & 2.5 & $203-235$ & 7 \\
\hline MCW69 & 55.7 & 1.0 & $160-180$ & 4 \\
\hline GUJ01 & 59.1 & 1.0 & $221-239$ & 5 \\
\hline GUJ13 & 55.7 & 1.5 & $145-159$ & 6 \\
\hline GUJ17 & 55.7 & 1.0 & $149-171$ & 9 \\
\hline GUJ59 & 55.7 & 1.0 & $200-248$ & 19 \\
\hline GUJ66 & 57.0 & 1.0 & $164-240$ & 36 \\
\hline GUJ84 & 55.7 & 1.0 & $181-185$ & 6 \\
\hline GUJ86 & 58.3 & 2.0 & $100-114$ & 5 \\
\hline NMG13 & 56.0 & 1.5 & $194-198$ & 4 \\
\hline NMG17 & 56.0 & 1.5 & 3 \\
\hline
\end{tabular}

